ARTICLE

https://doi.org/10.1038/s41467-019-11787-5

\title{
Diversity-triggered deterministic bacterial assembly constrains community functions
}

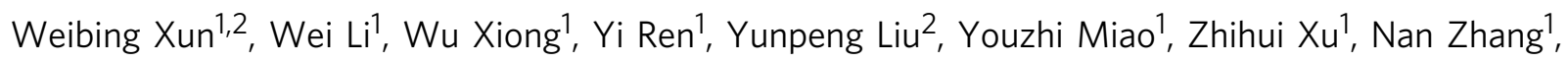
Qirong Shen ${ }^{1} \&$ Ruifu Zhang,

A growing body of evidence suggests that microbial $\alpha$-diversity (local species richness) may have positive effects on ecosystem function. However, less attention has been paid to $\beta$-diversity (the variation among local microbial assemblages). Here we studied the impact of microbial $\alpha$-diversity on stochastic/deterministic microbial community assembly processes, which are related to $\beta$-diversity, and the consequences for community function. Bacterial communities differing in $\alpha$-diversity were generated and their structures and potential community functional traits were inferred from DNA sequencing. Phylogenetic null modeling analysis suggests that stochastic assembly processes are dominant in high-diversity communities. However, in low-diversity communities, deterministic assembly processes are dominant, associating with the reduction of specialized functions that are correlated with specific bacterial taxa. Overall, we suggest that the low-diversity-induced deterministic community assembly processes may constrain community functions, highlighting the potential roles of specialized functions in community assembly and in generating and sustaining the function of soil ecosystems.

\footnotetext{
${ }^{1}$ Jiangsu Provincial Key Lab of Solid Organic Waste Utilization, Jiangsu Collaborative Innovation Center of Solid Organic Wastes, Educational Ministry Engineering Center of Resource-Saving Fertilizers, Nanjing Agricultural University, Nanjing 210095, China. ${ }^{2}$ Key Laboratory of Microbial Resources Collection and Preservation, Ministry of Agriculture, Institute of Agricultural Resources and Regional Planning, Chinese Academy of Agricultural Sciences, Beijing 100081, China. Correspondence and requests for materials should be addressed to Q.S. (email: shenqirong@njau.edu.cn) or to R.Z. (email: rfzhang@njau.edu. $\mathrm{cn})$
} 
$\mathrm{M}$ icrobial communities are the most diverse and dominant groups of organisms in terrestrial ecosystems. The activity of microorganisms greatly influence a variety of ecosystem functions and contribute to soil productivity and nutrient cycling, as well as many other ecosystem properties and services $^{1}$. One major general hypothesis states that ecosystems containing numerous species exhibit high levels of ecosystem functions and are functionally stable ${ }^{2}$. This prediction assumes that species overlap can sufficiently provide some redundancy and buffer ecosystem functions against biodiversity $\operatorname{loss}^{3}$. An extensive body of theoretical, experimental, and observational studies across different types of ecosystems confirm that high level of local diversity is important for generating and sustaining ecosystem functions and services ${ }^{4-6}$. Thereby, a dramatic loss of biodiversity has significant adverse impacts on ecosystem functions $^{7}$. However, other attempts to study the relationships between biodiversity and ecosystem function have yielded mixed results $^{8-12}$. Therefore, although high levels of bacterial diversity have led to the assumption that some bacteria are functionally redundant, we know little concerning how the interactions within diversity-related bacterial communities mediate ecosystem function and community assembly.

Various driving factors of microbial diversity have been intensively investigated across different spatial and temporal scales $^{13-15}$. Environmental surveys demonstrate that soil bacterial diversity can be strongly affected by $\mathrm{pH}$, soil type, latitude, vegetation, moisture, temperature, and nutrient availability. Among these, soil $\mathrm{pH}$ is the best predictor of both soil bacterial diversity and richness ${ }^{16}$, whereas soil type strongly influences soil bacterial composition ${ }^{17}$. Although the general patterns underlying variations in biodiversity have been observed, the factors controlling these patterns remain unclear. In general, the generation of soil microbial diversity and function is referred to as the community assembly processes. Microbial community assembly, which reflects the aggregation of spatiotemporal processes $^{18}$ that determine community composition, falls into two predominant categories that can be summarized as deterministic and stochastic processes ${ }^{19}$. Regardless of which of these two processes is dominant, community assembly determines the presence and abundance of species. According to Wardle and Putten $^{20}$, the species abundance is also an important determinant of ecosystem functions and the identity of those species. Therefore, the assembly process can necessarily influence soil microbial diversity and composition, with downstream impacts on the function of the system ${ }^{21}$. Mori et al. ${ }^{22}$ recently point out that $\beta$ diversity, species turnover between two sites or communities, is especially important in the context of ecosystem multifunctionality. However, much less attention has been paid to $\beta$ diversity than to $\alpha$-diversity. $\beta$-diversity is useful for inferring the stochastic and deterministic processes of community assembly for soil microbial communities ${ }^{23}$. Therefore, deeper knowledge of soil microbial $\beta$-diversity and the linkage between diversity and function will lead to a better understanding of the contributions of biodiversity to functional processes.

In this study, we establish a soil microcosm incubation experiment by inoculating progressively diluted soil suspensions into sterilized and $\mathrm{pH}$-amended soils, to clarify the uncertain relationship between $\alpha$-diversity and assembly processes (stochastic/deterministic processes related to $\beta$-diversity), and to assess the role of assembly processes in generating community function. We present the results of taxonomic features and potential community functional traits of the re-assembled bacterial communities from DNA sequencing and find that the reduced specialized functions dominate the deterministic community assembly processes, laying a foundation for deciphering the linkages between biodiversity and community functions.

\section{Results}

Taxonomic features of the re-assembled bacterial community. Dilution had significant impacts on the composition and $\alpha$-diversity of the re-assembled bacterial communities (Fig. 1a, b and Supplementary Fig. 1). The enriched bacterial groups related to high dilution levels were significantly ( $P$-value $<0.001$, one-way analysis of variance (ANOVA)) over-represented in the phyla/class of Betaproteobacteria, Gammaproteobacteria, and Bacteroidetes, whereas the depleted bacterial groups were mostly affiliated with Acidobacteria, Actinobacteria, Nitrospira, and Deltaproteobacteria. Bacterial $\alpha$-diversity, as expressed by the Shannon diversity index, was the highest in the initial soils and decreased along the dilution gradient (Fig. 1b). Although the suspensions were the same when inoculated into the two types of $\mathrm{pH}$-amended soils, bacterial $\alpha$ diversity peaked at neutral $\mathrm{pH}$. However, these diversity indices were less variable across $\mathrm{pH}$ levels than across dilution levels. Nonmetric multidimensional scaling (NMDS) analysis based on Bray-Curtis dissimilarity (Fig. 1a) showed that the samples derived from black soil grouped separately from the samples derived from red soil on the horizontal axis. Moreover, the samples across the soil $\mathrm{pH}$ gradient could be distinguished on the vertical axis, with samples being more dispersed at higher dilution levels.

Taxonomic abundance-based Weighted UniFrac community dissimilarity between different $\mathrm{pH}$ levels showed significantly larger variances within the more diluted treatments for both black and red soils (Fig. 2a), indicating larger variances within the communities that exhibited lower $\alpha$-diversity. Large variances between the two types of soils were observed at every dilution level (Fig. 2b), although these variances became smaller within more diluted samples at the same $\mathrm{pH}$ level (Supplementary Fig. 2).

Although soil type and $\mathrm{pH}$ had less significant impacts on soil bacterial $\alpha$-diversity than dilution, they nevertheless affected the structure of re-assembled bacterial community. The relative contributions of soil type, soil $\mathrm{pH}$, and the concentrations of $\mathrm{Ca}$, $\mathrm{Fe}$, and $\mathrm{SO}_{4}{ }^{2-}$ on the community variances were evaluated using variation partitioning analysis (VPA) for each dilution level (Fig. 2c). The contribution of soil type to the community variances decreased from $46.2 \%$ ( $P$-value $<0.001$ based on partial mantel test) in the initial soils (Fig. 2d) and $47.9 \%(P$-value $<0.001$ based on partial mantel test) in the $10^{-1}$ diluted samples (Fig. 2e) over $40.2 \%(P$-value $<0.001$ based on partial mantel test $)$ in the $10^{-4}$ diluted samples (Fig. 2f) to $29.7 \%(P$-value $<0.001$ based on partial mantel test) in the $10^{-7}$ diluted samples (Fig. $2 \mathrm{~g}$ ) and $19.3 \%$ ( $P$-value $<0.001$ based on partial mantel test $)$ in the most diluted samples (Fig. 2h). In contrast, the effect of soil pH increased along the dilution gradient $(9.6 \%, 10.7 \%, 13.6 \%, 28.1 \%$, and $43.3 \%$ of the variance in the initial soil and increasingly diluted samples, respectively; $P$-value $<0.001$ based on partial mantel test). Moreover, the concentrations of $\mathrm{Ca}$ and Fe had little impacts on the re-assembled communities, although different amounts of $\mathrm{CaO}$ and $\mathrm{FeSO}_{4}$ were used to manipulate soil $\mathrm{pH}$. Consistently, permutational multivariate ANOVA (PERMANOVA $)$ showed that soil type $\left(P\right.$-value $=0.001, F_{1,502}=16.73$ using PERMANOVA $)$ and $\mathrm{pH}\left(P\right.$-value $=0.001, F_{4,475}=7.02$ using PERMANOVA) altered the composition of bacterial communities significantly, explaining $35.2 \%$ and $29.5 \%$ of the variances, respectively. Meanwhile, the concentrations of $\mathrm{Ca}, \mathrm{Fe}$, and $\mathrm{SO}_{4}{ }^{2-}$ explained only $3.0 \%\left(P\right.$-value $=0.037, F_{9,470}=1.97$ using PERMANOVA), $2.4 \%\left(P\right.$-value $=0.063, F_{9,470}=1.49$ using PERMANOVA $)$, and $1.1 \%\left(P\right.$-value $=0.046, F_{9,470}=1.90$ using PERMANOVA) of the variances in bacterial community composition, respectively.

Assembly processes of the bacterial communities. To discriminate between the deterministic and stochastic processes in 


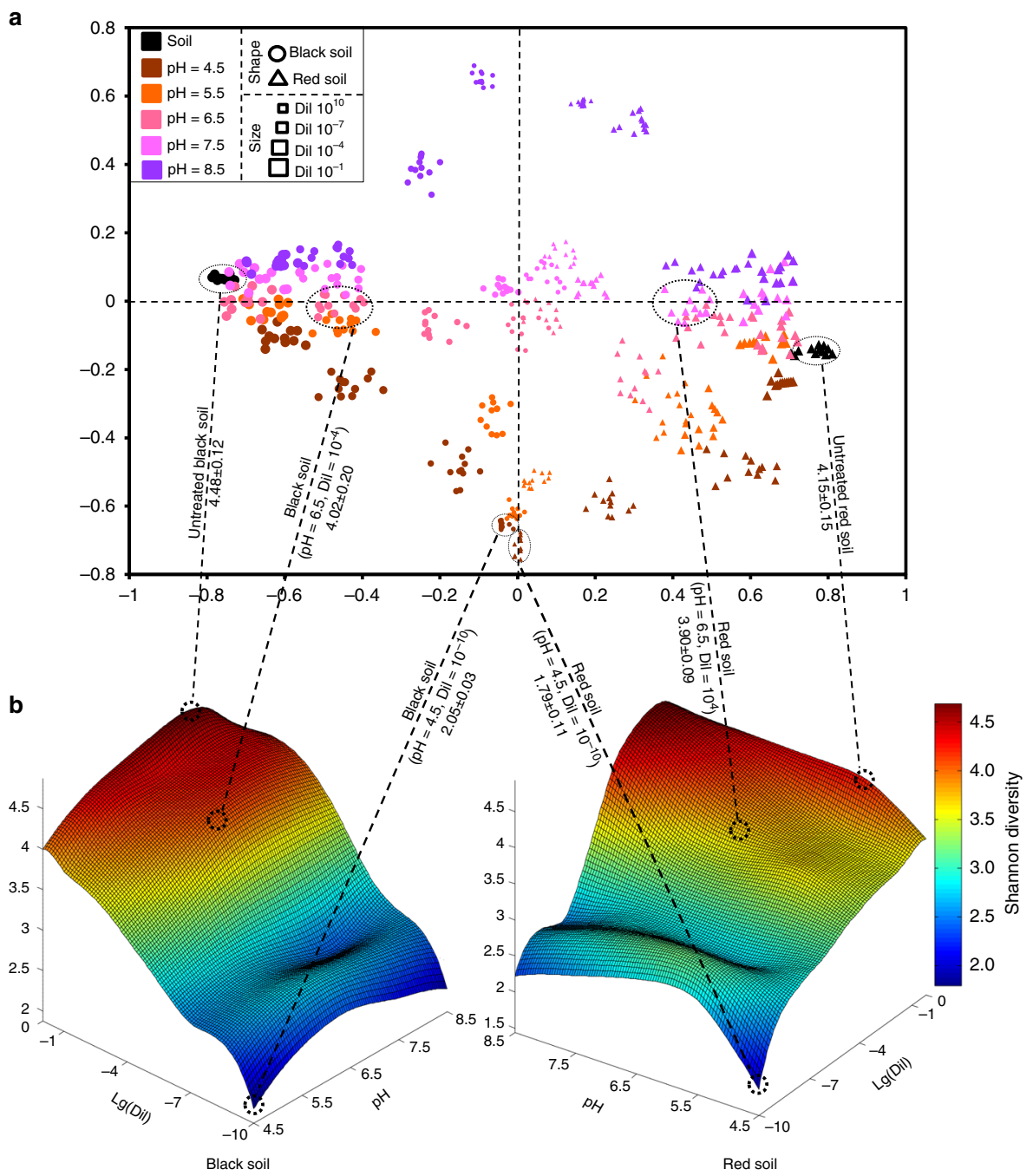

Fig. 1 Dimension reduction analysis and Shannon diversity of re-assembled bacterial communities. a Non-metric multidimensional scaling (NMDS) analysis of all incubated soil samples based on the Bray-Curtis taxonomic similarity. $\mathbf{b}$ The variances of Shannon diversity indices across soil pH and dilution gradients for black (left) and red (right) soils. Selected samples and corresponding diversity indices are connected by dotted lines. $\mathrm{Lg}$ (Dil) indicates the Lgtransformed dilution level. $\operatorname{Lg}(\mathrm{Dil})=0$ represents the untreated soil

community assembly along the dilution and $\mathrm{pH}$ gradients, we calculated the $\beta$-nearest taxon index $(\beta \mathrm{NTI})$ of every treatment. To infer alterations in the deterministic/stochastic assembly processes along dilution and $\mathrm{pH}$ gradients, we examined the relationships between $\beta$ NTI and dilution and $\mathrm{pH}$ gradients. The $\beta$ NTI values within each treatment showed various patterns related to $\mathrm{pH}$ and dilution level (Fig. 3). In both black and red soils, community assembly shifted from stochastic processes $(|\beta \mathrm{NTI}|<2)$ to deterministic processes $(|\beta \mathrm{NTI}|>2)$ with increasing dilution levels and the $\beta$ NTI values were significantly correlated with dilution gradients for all $\mathrm{pH}$ levels (Fig. 3a, b). However, the trends in $\beta$ NTI distribution over dilution gradients were not identical among different soil $\mathrm{pH}$ levels. Stochastic assembly processes were dominant in the least diluted samples and shifted towards dominance of variable selection $(\beta \mathrm{NTI}>2)$ at the $\mathrm{pH}$ value of 6.5 , while there was a shift towards dominance of homogeneous selection $(\beta \mathrm{NTI}<-2)$ under more acidic $(\mathrm{pH} 4.5)$ and alkaline ( $\mathrm{pH} 8.5)$ conditions in more diluted samples.

The same $\beta$ NTI datasets were reorganized to examine $\beta$ NTI distributions across the $\mathrm{pH}$ gradient from 4.5 to 8.5 for each dilution level. For both the black and red soils, the $\beta$ NTI values exhibited unimodal patterns along the $\mathrm{pH}$ gradient (Fig. $3 \mathrm{c}, \mathrm{d}$ ). Peak values were observed for neutral $\mathrm{pH}$ soils, whereas valley values were observed under acidic and alkaline conditions. In addition, the magnitude of these patterns increased with higher dilution levels. These results suggested that a bacterial community with lower species richness was more likely to follow deterministic assembly processes. Lastly, all $\beta$ NTI values were combined, resulting in a significantly negative relationship between $|\beta N T I|$ and soil bacterial Shannon diversity indices (Spearman's correlation coefficient $R^{2}=0.38, P$-value $<0.001$, two-sided tests; Fig. 3e).

Potential functions of the re-assembled bacterial community. To assess how soil $\mathrm{pH}$ and dilution affect the potential functions of the re-assembled bacterial communities, shotgun metagenomic sequencing and analyses were conducted on the DNA extractions from the red soil samples. We compared substance metabolismrelated genes, most of which are related to carbon, nitrogen, and sulfur turnover, at various $\mathrm{pH}$ and dilution levels (Fig. 4a). These functional categories were chosen, as many ecological services, e.g., food production and pollutant degradation and purification, rely largely on microbial functions related to the metabolism of varying substances. The relative abundances of every functional category differed significantly among dilution levels but less significantly, or in some cases not at all, across different $\mathrm{pH}$ levels. 
a

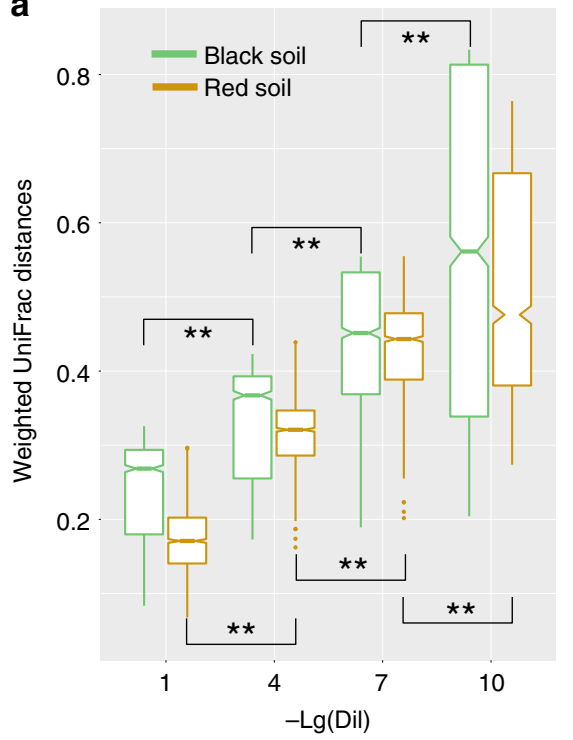

b

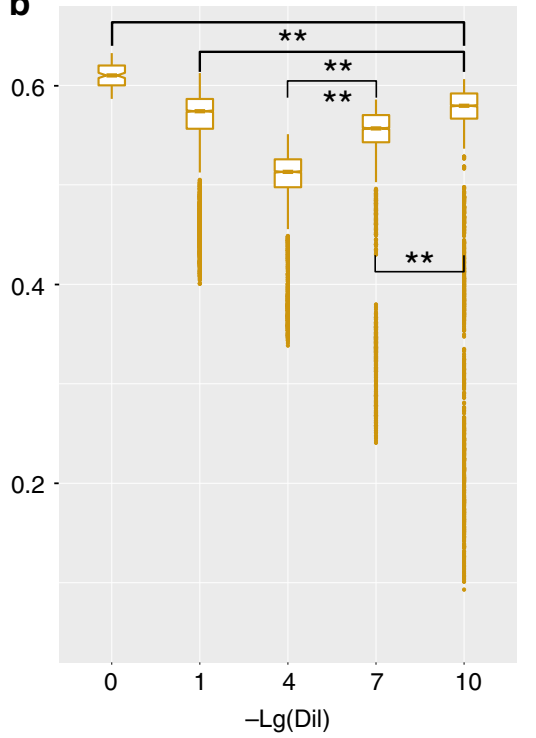

C

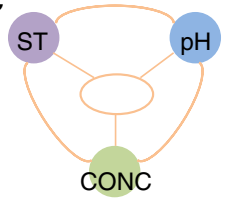

d

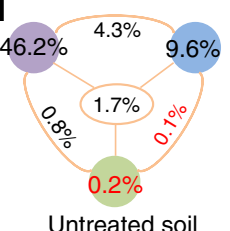

Untreated soil

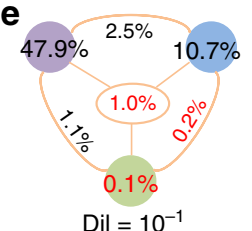

f

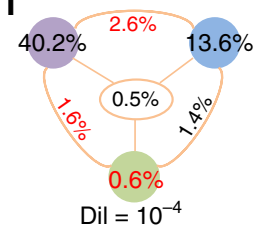

g

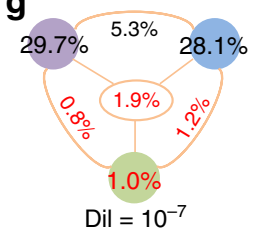

h

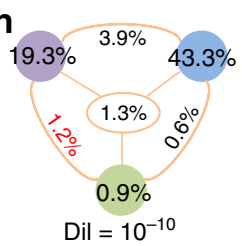

Fig. 2 Bacterial community variations between and within soil types. a Weighted Unifrac distances of pairwise bacterial communities from different pH levels within black and red soils, respectively, at each dilution level. b Weighted Unifrac distances of pairwise bacterial communities at different dilution levels between black and red soil samples. $\operatorname{Lg}(\mathrm{Dil})$ indicates the $\mathrm{Lg}$-transformed dilution level. $\operatorname{Lg}(\mathrm{Dil})=0$ represents the untreated soil. Asterisks indicate significance: ${ }^{* *} P$-value $<0.01$ based on Tukey's HSD test. Boxplot: median, 25\%/75\% percentiles, and the highest, lowest, and extremely values are shown. c Variation partitioning analysis (VPA) among soil type (ST), soil pH (pH), and the concentrations of $\mathrm{Ca}$ and Fe (CONC) for $\mathbf{d}$ untreated soil, and $\mathbf{e} 10^{-1}$, $\mathbf{f}$ $10^{-4}, \mathbf{g ~} 10^{-7}$, and $\mathbf{h} 10^{-10}$ diluted samples. Numbers indicate the percentage of variations in the bacterial community. Black numbers indicate significant variances ( $P$-value $<0.01$ based on Mantel test). Red numbers indicate variances that are not significant
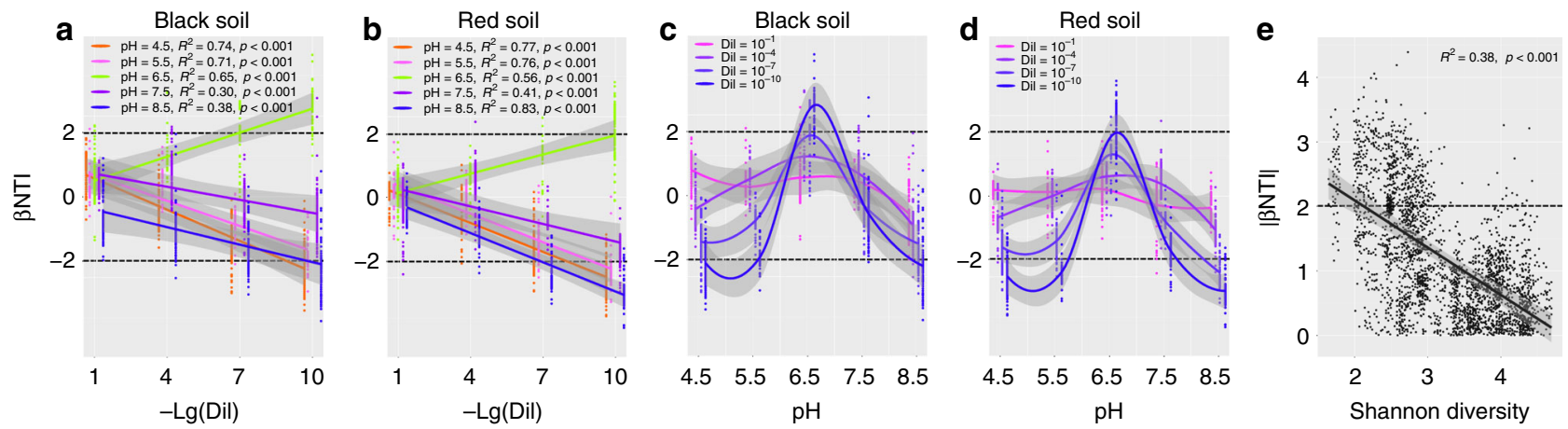

Fig. $3 \beta N T I$ values are associated with dilution and $\mathrm{pH}$ levels, and bacterial Shannon diversity. a The relationships between $-\mathrm{Lg}(\mathrm{Dil})$ and $\beta \mathrm{NTI}$ in black soil. b The relationships between $-\mathrm{Lg}(\mathrm{Dil})$ and $\beta N T I$ in red soil. c The effect of soil pH on $\beta N T I$ in black soil. $\mathbf{d}$ The effect of soil pH on $\beta N T I$ in red soil. e The relationship between $|\beta N T I|$ and bacterial Shannon diversity indices. Dil indicates the dilution level. Lg(Dil) indicates the Lg-transformed dilution level. Lg $(\mathrm{Dil})=0$ represents the untreated soil

A function carried out by narrow groups of microorganisms, meaning the related genes are distributed in specific microorganisms, will become gradually less abundant in samples seeded with more diluted inocula. For instance, the overall functions (specialized functions defined as functional group 1, FunGp1 hereafter) related to "Sulfur metabolism," "Nitrogen metabolism," "Methane metabolism," "Terpenoids \& Polyketides metabolism," and "Xenobiotics biodegradation and metabolism" (including "Atrazine degradation" and "Polycyclic aromatic hydrocarbon degradation," etc.) were gradually eliminated as the dilution increased (Fig. 4a). Correlation analysis of all the unique genes within these categories demonstrated that large proportions $(31.45-64.87 \%)$ of these genes were significantly decreased in relative abundances along the dilution level, whereas only small proportions (1.67-9.22\%) of these genes were significantly increased (Supplementary Table 1). Among these genes, indicator analysis (IndVal) showed that a specific gene of FunGp1, the periplasmic nitrate reductase gene napA of nitrogen metabolism
(IndVal $=0.938, P$-value $=0.001$ using the multipatt function of IndVal), was enriched as an indicator gene in more diluted samples, whereas other genes, such as phosphoadenosine phosphosulfate reductase gene $c y s H$ of sulfur metabolism (IndVal $=0.946, P$-value $=0.002$ using the multipatt function of IndVal) were depleted by dilution (Supplementary Table 2).

In contrast, other functions (broad functions defined as functional group 2, FunGp2 hereafter) related to "Glycolysis/ Gluconeogenesis," "TCA cycle," and the metabolism of some readily usable carbohydrates such as fructose, galactose, starch, and sucrose, were more presented in more diluted samples (Fig. 4a). For these functional categories of FunGp2, large proportions (34.89-61.95\%) of these genes were significantly increased with dilution level, whereas only small proportions $(1.98-8.12 \%)$ of these genes were significantly decreased (Supplementary Table 1). Indicator analysis showed that the metabolic genes of some essential amino acids (phenylalanine and tryptophan) were depleted, whereas the metabolic genes of some non-essential 
a
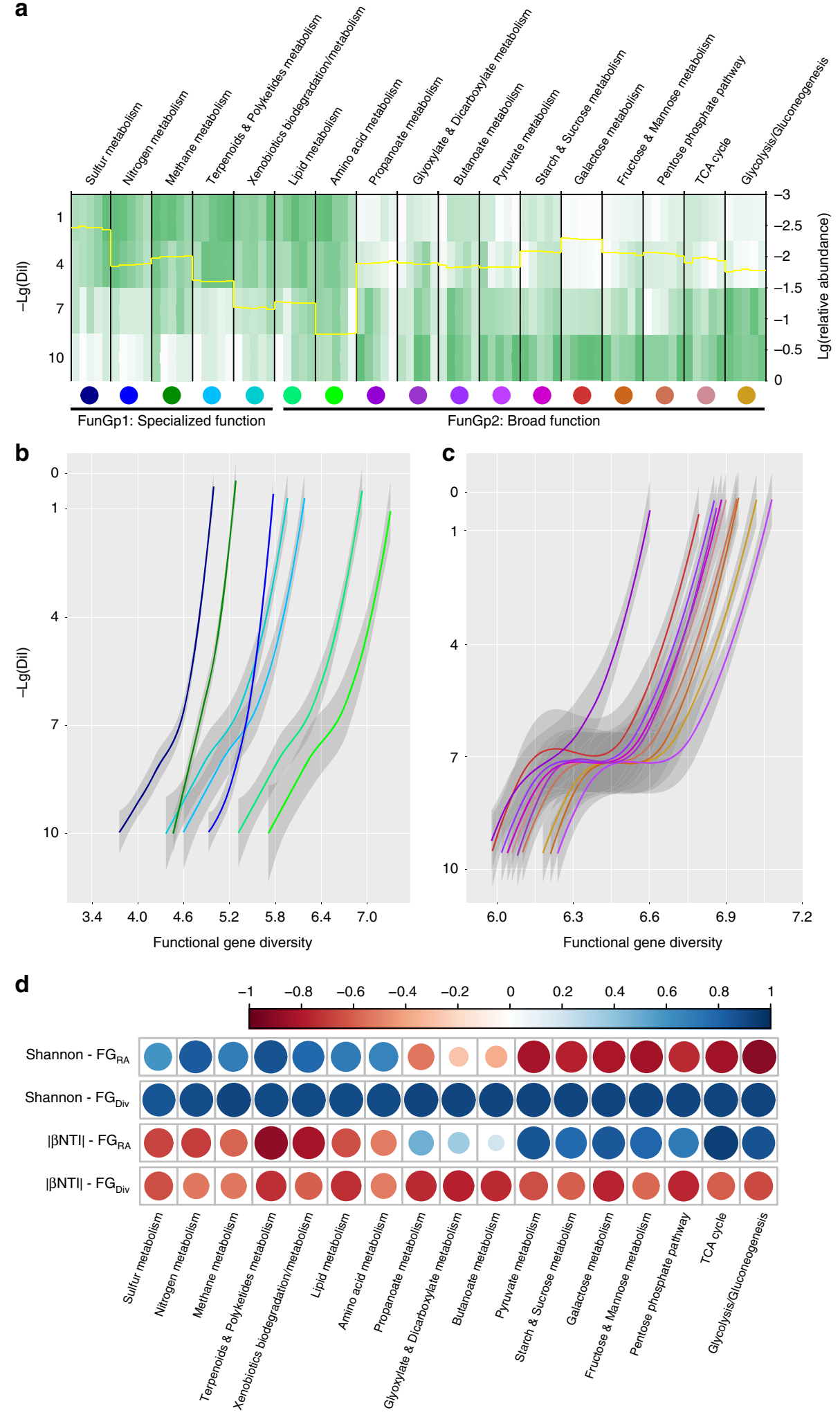

Fig. 4 Functional genes differ in abundance and diversity among treatments. a Heatmap of the relative abundances of different functional categories based on the normalized metagenomic data in $10^{-1}, 10^{-4}, 10^{-7}$, and 10-10 diluted samples. Color scaled from highest (green) to lowest (white) relative abundances within functional categories. Five columns of each functional category in heatmap indicate the soil pH range from 8.5 (left) to 4.5 (right). Yellow solid line indicates the Lg-transformed relative abundances of various functional categories. $\mathbf{b}$ The effect of dilution on specialized functional gene diversity. $\mathbf{c}$ The effect of dilution on broad functional gene diversity. The color lines in $\mathbf{b}$ and $\mathbf{c}$ represent different functional categories indicated by the color points in a. $\mathrm{Lg}(\mathrm{Dil})$ indicates the Lg-transformed dilution level. $\mathrm{Lg}(\mathrm{Dil})=0$ represents the untreated soil. $\mathbf{d}$ Correlations between Shannon and $\mathrm{FG}_{\mathrm{RA}}$

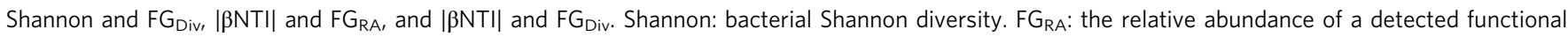
category. $\mathrm{FG}_{\mathrm{Div}}$ : the diversity of a detected functional category. The color points in $\mathbf{d}$ indicate the correlation coefficient (bluer indicates stronger positive correlation and redder indicates stronger negative correlation) and significance (larger size indicates lower $P$-value) 
amino acids (histidine) were enriched under dilution (Supplementary Table 2). However, not all functional categories in FunGp2 follow this trend; the "Amino acid metabolism" and "Lipid metabolism" functions showed a general depletion trend by dilution with $36.76 \%$ and $30.09 \%$ of significant decreased genes, respectively (Fig. 4a and Supplementary Table 1).

Despite of these opposing patterns on the relative abundances of FunGp1 and FunGp2, the diversity of all functional categories followed a decreasing pattern with increasing dilution level (Fig. $4 \mathrm{~b}, \mathrm{c})$. Moreover, the decreasing amplitudes of functional diversity were greater for FunGp1 (Fig. 4b) when compared with FunGp2 (Fig. 4c). To further investigate the impact of bacterial diversity and deterministic/stochastic assembly processes on the relative abundance $\left(\mathrm{FG}_{\mathrm{RA}}\right)$ and diversity $\left(\mathrm{FG}_{\mathrm{Div}}\right)$ of metabolic functions, we calculated the correlations between $|\beta N T I|$ values and their corresponding Shannon diversity indices, $F_{\mathrm{RA}}$ values, and $\mathrm{FG}_{\mathrm{Div}}$ values (Fig. $4 \mathrm{~d}$ ). The diversity and relative abundance of functional traits belonging to FunGp1, as well as the diversity of functional traits belonging to FunGp2, were positively correlated with Shannon diversity and negatively correlated with $|\beta N T I|$ values. In contrast, the relative abundances of functional traits belonging to FunGp2 were negatively correlated with Shannon diversity, but positively correlated with $|\beta \mathrm{NTI}|$ values. Therefore, FunGp1 and FunGp2 responded differently to bacterial diversity loss and may contribute differently to bacterial community assembly.

\section{Discussion}

The method used in this study was based on the inoculation of serially diluted soil suspensions into $\gamma$-irradiated sterilized and $\mathrm{pH}$-amended soil samples, to manipulate the bacterial species richness of the re-assembled microbiome after microcosm incubation $^{24,25}$. Our objective was to assess the importance of bacterial diversity-related functional gene pool in establishing a community assemblage. In the samples with the same dilution level, we only noticed a moderate shift in the bacterial species richness across soil $\mathrm{pH}$ levels. Shannon diversity indices peaked at neutral $\mathrm{pH}$ and were similar or, in some cases, significantly higher in black soil than in red soil. These differences were likely due to the direct impact of $\mathrm{pH}$ on community diversity ${ }^{16}$ and might be related to the higher nutrient availability in black soil ${ }^{26}$. These relatively moderate shifts in bacterial diversity of re-assembled microbiome correlated to soil type and $\mathrm{pH}$ were contrasted to the much stronger dilution effects. Therefore, dilution reduced the bacterial diversity in the soil suspensions used as inocula ${ }^{24}$ and subsequently affected the bacterial diversity in the re-assembled soil microbial communities, which confirmed the results of previous studies ${ }^{25}$.

We observed consistently significant positive correlations between bacterial $\alpha$-diversity and functional gene diversity. Functional gene categories of FunGp1 and FunGp2 responded to dilution differently. The differences were particularly striking for the genes encoding functions related to "Sulfur metabolism," "Nitrogen metabolism," "Methane metabolism," "Terpenoids \& Polyketides metabolism," and "Xenobiotics biodegradation and metabolism" of FunGp1, which were depleted upon dilution, although a small proportions of these genes were not. The influence of taxonomic diversity decline on the functional genes with increasing dilution may be explained by the lack of taxonomic redundancy for these specialized functions ${ }^{27}$. Due to their high dependency on narrowly distributed physiological pathways $^{28}$, these specialized functions are generally assumed to be highly dependent on environmental conditions ${ }^{29}$. Due to their scarcities in most soil microbiome, these specific functions would be particularly affected by dilution, as they are more likely to be removed over more abundant taxa. Numerous studies have suggested that some bacterial members of Acidobacteria, Actinobacteria, and Nitrospira are keystone taxa, which may harbor specialized functions such as nitrogen fixation or ammonia oxidation $^{30-32}$. For instance, the gene clusters inferring to "Terpenoids and Polyketides metabolism" are usually harbored by a minority of soil microbial community, such as some species of Actinobacteria $^{33}$. Several investigations have demonstrated that the enzymes involved in "Xenobiotics biodegradation/metabolism" are inducible and only exist in a small group of microorganisms ${ }^{34,35}$. Consistently, the taxonomic composition results in our study demonstrated that the bacterial groups of Acidobacteria, Actinobacteria, and Nitrospira were depleted in more diluted samples. Thus, the dilution-induced loss of rare species may significantly constrain the physiological pathways of specialized functions and result in a weakened metabolic network.

The functions of FunGp2, related to "Glycolysis/Gluconeogenesis," "TCA cycle," and "Pentose phosphate pathway" are critical for microbial growth and propagation. These functions, the so-called broad functions ${ }^{27}$, are widely distributed among living organisms and were enriched by dilution. Among these broad functions, the "Amino acid metabolism" and "Lipid metabolism" functions are quite widely distributed. For instance, the biosynthesis of iso- and anteiso-fatty acids occurs in many bacteria, as the major acyl constituents of membrane lipids ${ }^{36}$ and the biosynthesis pathways of non-essential amino acids ${ }^{37}$ are distributed broadly among diverse bacteria. However, these two functions were generally depleted by dilution. We inferred that the biosynthesis of omega-cyclohexyl and omega-cycloheptyl fatty acids ${ }^{36}$, and the metabolisms of some essential amino acids ${ }^{38}$ are only present in small populations of bacterial species. Consequently, the "Amino acid metabolism" and "Lipid metabolism" are functions in between the specialized and broad functions. Moreover, we observed that the diversity of these two functional groups decreased with increasing dilution levels. Besides, the decreasing amplitudes in FunGp2 were smaller than those in FunGp1. These observations suggested that the broad functions were more redundant than the specialized functions and the overlap of the functional capabilities of species in a community may follow different subtractive shapes ${ }^{39}$.

Functional gene diversity is an important indicator for functional redundancy, whereas gene abundance may represent functional capacity. Soil microbial diversity loss results in a significant decrease in specialized functional capacity, such as potential denitrification activity 40 and pesticide mineralization capacity ${ }^{34}$. In contrast, with the inoculation of gradient-diluted soil suspensions in an established microcosm incubation experiment, Griffiths et al. ${ }^{12}$ found there were no differences in soil respiration, which is a broad function, although the microbial diversity decreased with intensified dilution. Therefore, we suggest that the decreasing of specialized functions synchronously resulted in increased proportions of broad functions and, hence, broad functions were more abundant in more diluted samples.

The relationships between decreases in functional gene diversity and the degradation of ecosystem function and service have been widely investigated based on $\alpha$-diversity, but particularly lacking on $\beta$-diversity ${ }^{22}$. Considering there is no ubiquitous species assemblage that can support all functions simultaneously, community functions require different sets of species assemblages under various environmental conditions ${ }^{41}$. Moreover, the stochastic/deterministic processes to form species assemblages maybe well-defined by $\beta$-diversity ${ }^{23}$. Consequently, $\beta$-diversityrelated analyses should be considered in studies investigating relationships between biodiversity and ecosystem function.

In this study, for each type of soil, the bacterial communities from less diluted samples were more similar than those from 
more diluted samples. Dilution reduced species richness, leading to decreased resilience and increased sensitivity to soil $\mathrm{pH}$ changes. As community stability is enhanced in species-rich communities ${ }^{42-44}$, a community with lower species richness will be more susceptible to environmental changes, e.g., soil $\mathrm{pH}$ changes. Not surprisingly, the VPA results demonstrated that the effect of soil type on the re-assembled bacterial communities decreased, whereas the effect of $\mathrm{pH}$ increased with increasing dilution levels. However, we observed few significant differences in the abundances of every functional category among soil $\mathrm{pH}$ levels, suggesting that the community functional gene composition may not be constrained by taxonomy ${ }^{45}$.

Our results showing the shift patterns of $\beta$ NTI values through different $\mathrm{pH}$ and dilution levels clearly indicate that the community assembly tends to be dominated by deterministic processes when the taxonomic diversity level is low. This tendency was further confirmed by calculating the relationships between | $\beta \mathrm{NTI} \mid$ values and bacterial Shannon diversity indices. Previous studies ${ }^{46,47}$ have suggested that the community is more susceptible to drift (stochastic process) or founder effects with lower biomass and smaller population. This is true at the period of low population or community size $\mathrm{f}^{48}$. However, in an established community with saturated population or community size, the local dominance of stochastic or deterministic processes can be strongly affected by dispersal, which depends on the local environment ${ }^{49}$. In addition, we found the deterministic processes were correlated with decreased functional diversity and relative abundances of specialized functions. Hence, we suggest that dispersal in low-diversity communities may enhance environmental selection and contribute to deterministic (selection) processes. Therefore, there is a transition in bacterial community assembly from stochastic to deterministic processes with the decreasing of soil bacterial species richness and functional diversity. Moreover, according to the $\beta$ NTI values, deterministic processes tend to be a variable selection $(\beta \mathrm{NTI}>2)$ at neutral $\mathrm{pH}$ and a homogeneous selection $(\beta \mathrm{NTI}<-2)$ under acidic and alkaline conditions.

Taken together, this study has collectively visualized the structural and functional assembly patterns of bacterial communities on broad species richness across soil $\mathrm{pH}$ values in two types of soils. We therefore integrated these results into a conceptual model on how diversity-related stochastic/deterministic processes contribute to the structural and functional assembly of soil bacterial communities under various environmental conditions (Fig. 5). We assumed a re-assembled community in which the relative influences of stochastic/deterministic ecological processes become relatively stable in this framework. However, these stable processes may not exist in real ecosystems due to changing environments. We also did not take into account of the initial stage of community establishment, which is primarily dominated by stochastic processes ${ }^{50}$. However, in the later stages of assembly, microorganisms need to cope with two main stressors: environmental selection and species competition ${ }^{51}$.

Part I: The assembly of a high-diversity community. In this stage, community assembly is probably driven by comprehensive biotic and abiotic impacts ${ }^{52}$. Due to the high level of bacterial diversity, assembly is expected to involve complex biological interactions ${ }^{53,54}$ and nutrient availability is responsible for the intensity of these interactions ${ }^{55}$. Soil contains large amounts of complex and recalcitrant substrates that cannot be efficiently utilized by most microbes ${ }^{56}$ and the competition will intensify when available nutrients in the environment are insufficient to support a large number of microbes ${ }^{57}$. Consequently, specialized functions, including "Sulfur metabolism," "Nitrogen metabolism," etc., can maintain nutrient turnover in the soil and provide available nutrients for other microorganisms, thus potentially weakening the biotic competition. Moreover, in some extreme environments, some bacteria can interact with phagocytic cells in response to oxidative stress ${ }^{58}$ and some can metabolize pesticides (those possessing the "Xenobiotics biodegradation and metabolism" function) or immobilize heavy metal ions to reduce their toxic effects ${ }^{34,59}$. Therefore, these specialized functions play important roles in ecosystems that reduce environmental stress and increase the survival probability of other microorganisms that do not have these functions. Overall, diverse specialized functional groups with irreplaceable functions in nutrient cycling and inhibitor suppression are crucial in community assembly processes. Therefore, community structures are more likely to be independent of species traits and assembly processes are more likely to be stochastic processes related to birth, death, colonization, extinction, and speciation ${ }^{60}$.

Part II: The assembly of a low-diversity community in neutral $\mathrm{pH}$ soils. Soil $\mathrm{pH}$ can strongly influence bacterial community composition ${ }^{16}$ and a broad range of species can grow successfully in neutral $\mathrm{pH}$ soils ${ }^{61}$; hence, the influence of abiotic habitat filtering decreases. However, diversity loss reduces specialized functions and induces a weakened metabolic network that may compromise nutrient availability and increase biotic competition. Therefore, in neutral $\mathrm{pH}$ soils, community assembly should be primarily driven by biological interactions (simple but strong) and selections in a heterogeneous environment (affecting only a small group of local microbes). Thus, the assembly processes are more likely to be variable selections.

Part III: The assembly of a low-diversity community in acidic and alkaline soils. When soil $\mathrm{pH}$ becomes increasingly extreme, an environmental filter is intensified under high or low $\mathrm{pH}$ conditions ${ }^{16,62}$. Thereby, homogeneous selection (affecting all microbes in an ecosystem simultaneously) increases without specialized functions.

Our results indicate that dilution affects the assembly processes and significantly reduces the taxonomic diversity and functional diversity of re-assembled bacterial communities. The relative abundances of specialized functional categories are decreased, whereas the relative abundances of broad functional categories are increased in low-diversity communities. This situation leads to the dominance of deterministic processes in the assembly of communities with low bacterial diversity. However, in highdiversity communities, the existence of specialized functions leads to the dominance of stochastic assembly processes. Therefore, specialized functions are potentially critical for establishing bacterial community and maintaining community functions. Further $\beta$-diversity-related investigations will improve our understanding of community assembly processes and the consequences of community functions, which will be essential to ensure the sustained provision of ecosystems.

\section{Methods}

Experimental design and microcosm incubation. Black soil and red soil samples were collected from Haerbin $\left(127^{\circ} 54^{\prime} \mathrm{E}, 46^{\circ} 28^{\prime} \mathrm{N}\right)$ in Heilongjiang Province of Northeast China and Yingtan $\left(116^{\circ} 94^{\prime} \mathrm{E}, 28^{\circ} 21^{\prime} \mathrm{N}\right)$ in Jiangxi Province of South China, respectively. The black soil is characterized as Calcaric Chernozems and the red soil is an example of a Ferralic Cambisol according to the FAO/UNESCO System of Soil Classification. Samples were obtained from the upper $20 \mathrm{~cm}$ of soil perennially covered by weeds. The black soil had a $\mathrm{pH}$ of 8.0 and contained $4.6 \%$ organic matter, $100.8 \mathrm{mg} \mathrm{kg}^{-1} \mathrm{~N}, 16.7 \mathrm{mg} \mathrm{kg}^{-1} \mathrm{P}$, and $90.5 \mathrm{mg} \mathrm{kg}^{-1} \mathrm{~K}$. The red soil had a $\mathrm{pH}$ of 5.3 and contained $1.6 \%$ organic matter, $53.9 \mathrm{mg} \mathrm{kg}^{-1} \mathrm{~N}, 0.95 \mathrm{mg} \mathrm{kg}^{-1}$ $\mathrm{P}$, and $61.5 \mathrm{mg} \mathrm{kg}^{-1} \mathrm{~K}$. Both soils were homogenized and sieved with a $2 \mathrm{~mm}$ sieve. A portion for preparing the inocula were temporarily stored at room temperature $\left(20^{\circ} \mathrm{C}\right)$ and maintained at constant moisture level (30\% of field capacity) in regularly aerated bags, whereas the rest were sterilized by $\gamma$-irradiation ( $>50 \mathrm{kGray}$ ) (Xiyue Radiation Technology Co., Ltd, NJ, China).

Soil microcosms were prepared as described by Xun et al. ${ }^{51}$. Each microcosm was constructed by placing $250 \mathrm{~g}$ of sterilized soil into a $500 \mathrm{ml}$ bottle. Sterile distilled water was added to maintain a constant moisture level of $45 \%$ of field capacity and the microcosms were pre-incubated at $20^{\circ} \mathrm{C}$ in the dark for 4 weeks before sterility test on agar plates. During this 4 -week pre-incubation period, lime 


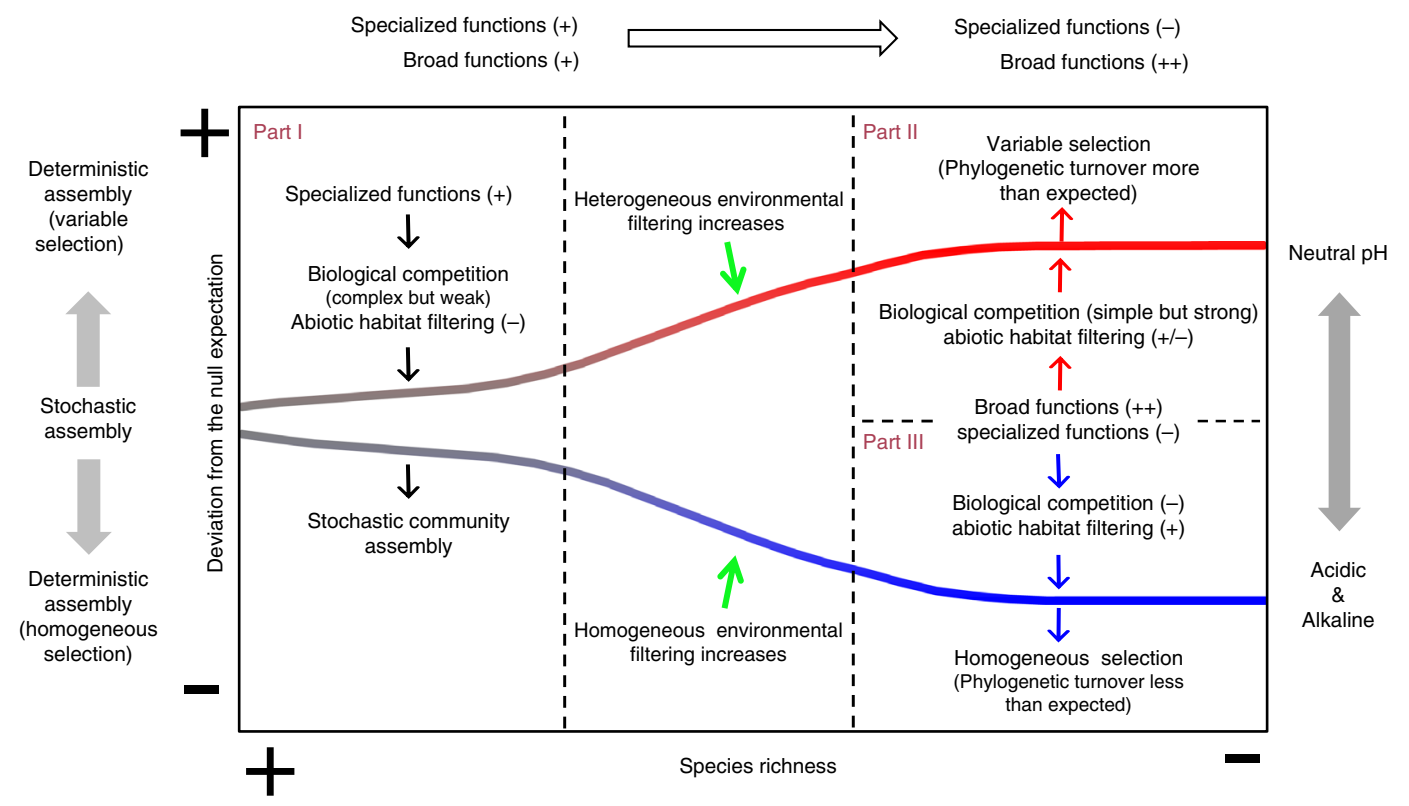

Fig. 5 Conceptual model of bacterial community assembly processes. This conceptual model shows how diversity-related stochastic/deterministic processes contribute to the structural and functional assembly of soil bacterial communities under various $\mathrm{pH}$ conditions

$(\mathrm{CaO})$ and ferrous sulfate $\left(\mathrm{FeSO}_{4}\right)$ were added (Supplementary Table 3) along with sterile distilled water to create a soil $\mathrm{pH}$ gradient $(4.5,5.5,6.5,7.5$, and 8.5$)$. The needed amount of $\mathrm{CaO}$ and $\mathrm{FeSO}_{4}$ were obtained based on a 1-month period soil incubation pre-experiment. The $10^{-1}$ soil suspension for inoculation was made by mixing $20 \mathrm{~g}$ of fresh soil ( $10 \mathrm{~g}$ of black soil and $10 \mathrm{~g}$ of red soil) in $180 \mathrm{ml}$ of sterile distilled water in a blender for $5 \mathrm{~min}$. This $10^{-1}$ suspension was then progressively diluted to create the $10^{-4}, 10^{-7}$, and $10^{-10}$ suspensions. These $10^{-1}, 10^{-4}, 10^{-7}$, and $10^{-10}$ suspensions were added to the microcosms of each $\mathrm{pH}$ level. We also included two untreated initial soils (untreated black and red soils but incubated as described for the other samples) that served as controls and all treatments were replicated six times. Therefore, we established a total of 252 microcosms $[(5 \mathrm{pH}$ levels $\times 4$ dilution levels $\times 2$ soil types +2 initial soils $) \times 6$ replicates]. All microcosms were then incubated at $20^{\circ} \mathrm{C}$ and $45 \%$ of field capacity in the dark and agitated regularly for 16 weeks. During the entire incubation period, the bottle mouth was covered by a semi-permeable membrane to allow air exchange and bottles were only opened in a sterile biological hood. The semi-permeable membrane was replaced after each opening. Soil $\mathrm{pH}$ was checked every 2 weeks and the concentrations of $\mathrm{Ca}, \mathrm{Fe}$, and $\mathrm{SO}_{4}{ }^{2-}$ were detected using standardized method of soil physico-chemical analysis (Supplementary Table 4).

DNA extraction and sequencing. After 16-week incubation, two replicate samples were collected from each microcosm. Total DNA was extracted from $0.25 \mathrm{~g}$ of incubated soil using the PowerSoil DNA Isolation Kit (Mo Bio Laboratories, Inc., Carlsbad, CA, USA). To minimize any DNA extraction bias, three successive DNA extractions of each soil sample were pooled before performing PCR reaction. A NanoDrop ND-2000 spectrophotometer (NanoDrop, ND-2000, Thermo Scientific, 111 Wilmington, DE, USA) was used to assess DNA quality according to the 260/ $280 \mathrm{~nm}$ and 260/230 $\mathrm{nm}$ absorbance ratios.

Amplification of the V4 hypervariable region of the bacterial $16 \mathrm{~S}$ rRNA gene was performed to assess the bacterial community using the primers $515 \mathrm{~F}: 5^{\prime}-\mathrm{G}$ TGCCAGCMGCCGCGGTAA-3' and 806 R: 5'-GGACTACHVGGGTWTCTAA T-3'. PCR amplifications were combined in equimolar ratios and sequenced on an Illumina MiSeq instrument. The sequencing data were processed using the UPARSE pipeline (http://drive5.com/usearch/manual/uparse_pipeline.html) ${ }^{63}$. Raw sequences were first subjected to quality control. Singleton and chimeric sequences were removed after dereplication. The remaining sequences were categorized into operational taxonomic units (OTUs) at $97 \%$ similarity and taxonomic assignment was performed using the Silva database (Release 128) (https://www.arb-silva.de/).

The DNA extractions from red soil samples for 16S rRNA gene amplicon sequencing were also used for metagenomic DNA sequencing on an Illumina HiSeq2000 platform (150 bp paired-end reads). Low-quality reads were filtered from the Illumina raw data based upon a minimum $Q$-score of 30 . All reads were assembled with IDBA 1.1.164 and further filtered using a minimum length of 150 bp. Finally, the assembly comprised $86,230,290$ contigs, with a total length of 61.75 $\mathrm{Gb}$. Genes were then predicted using MetaGeneMark $v 4.33^{65}$ and clustered with a 0.95 similarity threshold using CD-HIT v4.6.266. The number of reads per sample mapping to genes was calculated using SOAPaligner $2.21^{67} ; 39,317,785$ nonredundant unigenes were obtained (Supplementary Table 5). The Kyoto
Encyclopedia of Genes and Genomes (Version 58) database was used for functional gene annotation using the basic local alignment search tool.

Statistical analysis. For the $16 \mathrm{~S}$ rRNA gene amplicon sequencing data, a rarefied OTU table at 11,020 reads per sample was created according to the minimum number of sequences per sample (Supplementary Table 5). Relative abundance of one phylogenetic group was defined as the number of sequences affiliated with that group divided by the total number of sequences per sample. The Shannon diversity index ( $\alpha$-diversity) calculation and Bray-Curtis dissimilarity-based NMDS analysis were performed based on the rarefied OTU table. The VPA and PERMANOVA were used to determine the contributions of soil type and properties, including soil $\mathrm{pH}$ and the concentrations of $\mathrm{Ca}, \mathrm{Fe}$, and $\mathrm{SO}_{4}{ }^{2-}$, on the variation of bacterial community. The bacterial community data were normalized as percent frequency based on the rarefied OTU table and the concentrations of $\mathrm{Ca}$, Fe, and $\mathrm{SO}_{4}{ }^{2-}$ were normalized by min-max normalization for VPA and PERMANOVA. The bioevn command in the vegan R package (v.2.4-1) ${ }^{68}$ was used to select the best combination of environmental factors (best correlated with bacterial assemblage dissimilarity) and the adonis command in the vegan package was used to perform PERMANOVA. All the analyses above were performed using the vegan R package. The normalized percent frequency based on the rarefied OTU table was created to calculate the phylogenetic community dissimilarity ( $\beta$-diversity) using FastUnifrac ${ }^{69}$.

To infer the community assembly processes, we first calculated the mean nearest taxon distance metric using the picante $\mathrm{R}$ package ${ }^{70}$ and then implemented a previously developed null modeling approach to calculate the $\beta \mathrm{NTI}^{71,72}$. $\beta \mathrm{NTI}>$ 2 is considered indicative of significantly more than expected phylogenetic turnover, which is interpreted as variable selection of deterministic processes. $\beta N T I<-2$ indicates significantly less than expected phylogenetic turnover, which is interpreted as homogeneous selection of deterministic processes. If the $|\beta \mathrm{NTI}|<$ 2 , this indicates that the observed phylogenetic composition differences are the result of stochastic processes ${ }^{19}$.

For the metagenomic DNA-sequencing data, the percent frequency of one functional category was defined as the read count abundance affiliated with that category divided by the total read count abundance per sample. The functional gene diversity was calculated using the gene richness within each functional category. The normalized metagenomic data for heatmap to demonstrate functional gene enriched or depleted pattern within each functional category was normalized by removing the mean and dividing by the SD. To determine the characteristic functional genes that were enriched or depleted by dilution, the indicator analysis (IndVal) combining both the abundance and occurrence of a given gene was used. The IndVal values were calculated using the indicspecies $\mathrm{R}$ package (version 1.7.6) ${ }^{73}$. All the correlations were calculated using Spearman's correlations. Duncan's multiple comparisons test was used to calculate the significance among samples. Tukey's honestly significant difference test was used to calculate the significance between two samples. All statistical analyses were performed using R software (version 3.3.2).

Reporting summary. Further information on research design is available in the Nature Research Reporting Summary linked to this article. 


\section{Data availability}

The data supporting the findings of this study are available within the paper and its Supplementary Information files. The DNA sequences from all incubation samples are deposited in the NCBI Sequence Read Archive (SRA) database with accession numbers of SRR8857587, SRR8857588, SRR8857589, SRR8857590, SRR8857591, and SRR8840928.

\section{Code availability}

The "vegan", "picante", and "indicspecies" are packages for the R statistical language and environment. The codes for vegan (http://vegan.r-forge.r-project.org), picante (http:// picante.r-forge.r-project.org), and indicspecies (http://th.archive.ubuntu.com/cran/web/ packages/indicspecies/) are freely available on the web. The R codes used for calculating $\beta$ NTI metrics are provided in Supplementary Note 1.

Received: 23 August 2018 Accepted: 28 June 2019

Published online: 23 August 2019

\section{References}

1. Cardinale, B. J. et al. Biodiversity loss and its impact on humanity. Nature 489, 59-67 (2012)

2. Margalef, R. On certain unifying principles in ecology. Am. Nat. 97, 357-374 (1963).

3. Galand, P. E., Pereira, O., Hochart, C., Auguet, J. C. \& Debroas, D. A strong link between marine microbial community composition and function challenges the idea of functional redundancy. ISME J. 12, 2470-2478 (2018).

4. Tilman, D. et al. The influence of functional diversity and composition on ecosystem processes. Science 277, 1300-1302 (1997).

5. Naeem, S., Duffy, J. E. \& Zavaleta, E. The functions of biological diversity in an age of extinction. Science 336, 1401-1406 (2012).

6. Duffy, J. E., Godwin, C. M. \& Cardinale, B. J. Biodiversity effects in the wild are common and as strong as key drivers of productivity. Nature 549, 261-264 (2017).

7. Isbell, F. et al. Linking the influence and dependence of people on biodiversity across scales. Nature 546, 65-72 (2017).

8. Graham, E. B. et al. Microbes as engines of ecosystem function: when does community structure enhance predictions of ecosystem processes?. Front. Microbiol. 7, 214 (2016).

9. Graham, E. B. et al. Coupling spatiotemporal community assembly processes to changes in microbial metabolism. Front. Microbiol. 7, 1949 (2016)

10. Martiny, J. B., Jones, S. E., Lennon, J. T. \& Martiny, A. C. Microbiomes in light of traits: a phylogenetic perspective. Science 350, aac9323 (2015).

11. Bier, R. L. et al. Linking microbial community structure and microbial processes: an empirical and conceptual overview. FEMS Microbiol. Ecol. 91, fiv113 (2015)

12. Griffiths, B. S. et al. An examination of the biodiversity-ecosystem function relationship in arable soil microbial communities. Soil Biol. Biochem. 33, 1713-1722 (2001).

13. Locey, K. J. \& Lennon, J. T. Scaling laws predict global microbial diversity. Proc. Natl Acad. Sci. USA 113, 5970-5975 (2016)

14. Horner-Devine et al. A taxa-area relationship for bacteria. Nature 432 , 750-753 (2004).

15. Morlon, H. et al. Spatial patterns of phylogenetic diversity. Ecol. Lett. 14, 141-149 (2011).

16. Fierer, N. \& Jackson, R. B. The diversity and biogeography of soil bacterial communities. Proc. Natl Acad. Sci. USA 103, 626-631 (2006).

17. Lundberg, D. S. et al. Defining the core Arabidopsis thaliana root microbiome. Nature 488, 86-90 (2012).

18. Graham, E. B. et al. Deterministic influences exceed dispersal effects on hydrologically-connected microbiomes. Environ. Microbiol.19, 1552-1567 (2017).

19. Tripathi, B. M. et al. Soil pH mediates the balance between stochastic and deterministic assembly of bacteria. ISME J. 12, 1072-1083 (2018).

20. Wardle, D. A. \& Putten, W. H. V. D. Terrestrial ecosystem responses to species gains and losses. Science 332, 1273-1277 (2011).

21. Leibold, M. A., Chase, J. M. \& Ernest, S. K. Community assembly and the functioning of ecosystems: how metacommunity processes alter ecosystems attributes. Ecology 98, 909-919 (2016).

22. Mori, A. S., Isbell, F. \& Seidl, R. $\beta$-diversity, community assembly, and ecosystem functioning. Trends Ecol. Evol. 33, 549-564 (2018).

23. Chase, J. M. Stochastic community assembly causes higher biodiversity in more productive environments. Science 328, 1388-1391 (2010).

24. Yan, Y., Kuramae, E. E., Klinkhamer, P. G. L. \& Veen, J. Avan Revisiting the dilution procedure used to manipulate microbial biodiversity in terrestrial systems. Appl. Environ. Microbiol. 81, 4246-4252 (2015).
25. Yan, Y., Kuramae, E. E., Hollander, Mde, Klinkhamer, P. G. L. \& Veen, J. Avan Functional traits dominate the diversity-related selection of bacterial communities in the rhizosphere. ISME J. 11, 56-66 (2017).

26. Xun, W. et al. Significant alteration of soil bacterial communities and organic carbon decomposition by different long-term fertilization management conditions of extremely low-productivity arable soil in South China. Environ. Microbiol. 18, 1907-1917 (2016).

27. Delgado-Baquerizo, M., Grinyer, J., Reich, P. B. \& Singh, B. K. Relative importance of soil properties and microbial community for soil functionality: insights from a microbial swap experiment. Funct. Ecol. 30, 1862-1873 (2016).

28. Powell, J. R. et al. Deterministic processes vary during community assembly for ecologically dissimilar taxa. Nat. Commun. 6, 8444 (2015).

29. Rivett, D. W. \& Bell, T. Abundance determines the functional role of bacterial phylotypes in complex communities. Nat. Microbiol. 3, 767-772 (2018).

30. Ye, D. et al. Molecular ecological network analyses. BMC Bioinformatics 13, 113 (2012).

31. Chao, Y. et al. Structure, variation, and co-occurrence of soil microbial communities in abandoned sites of a rare earth elements mine. Environ. Sci. Technol. 50, 11481-11490 (2016).

32. Banerjee, S., Schlaeppi, K. \& van der Heijden, M. G. A. Keystone taxa as drivers of microbiome structure and functioning. Nat. Rev. Microbiol. 16, 567-576 (2018)

33. Crits-Christoph, A., Diamond, S., Butterfield, C. N., Thomas, B. C. \& Banfield, J. F. Novel soil bacteria possess diverse genes for secondary metabolite biosynthesis. Nature 558, 440 (2018).

34. Singh, B. K. et al. Loss of microbial diversity in soils is coincident with reductions in some specialized functions. Environ. Microbiol. 16, 2408-2420 (2014).

35. Gianfreda, L. \& Rao, M. A. in Xenobiotics in the Soil Environment: Monitoring, Toxicity and Management (eds Hashmi, M. Z., Kumar, V. \& Varma, A.) 49, 153-169 (Springer International Publishing, 2017).

36. Kaneda, T. Iso- and anteiso-fatty acids in bacteria: biosynthesis, function, and taxonomic significance. Microbiol. Mol. Biol. Rev. 55, 288-302 (1991).

37. Min, B., Pelaschier, J. T., Graham, D. E., Tumbula-Hansen, D. \& Söll, D. Transfer RNA-dependent amino acid biosynthesis: an essential route to asparagine formation. Proc. Natl Acad. Sci. USA 99, 2678-2683 (2002).

38. Wendisch, V. F. (ed.) Amino acid biosynthesis-pathways, regulation and metabolic engineering. 5 (Springer Science \& Business Media, 2007).

39. Yachi, S. \& Loreau, M. Biodiversity and ecosystem productivity in a fluctuating environment: the insurance hypothesis. Proc. Natl Acad. Sci. USA 96, 1463-1468 (1999)

40. Philippot, L. et al. Loss in microbial diversity affects nitrogen cycling in soil. ISME J. 7, 1609-1619 (2013).

41. Hillebrand, H. \& Matthiessen, B. Biodiversity in a complex world: consolidation and progress in functional biodiversity research. Ecol. Lett. 12, 1405-1419 (2010).

42. Cruz-Martínez, K. et al. Despite strong seasonal responses, soil microbial consortia are more resilient to long-term changes in rainfall than overlying grassland. ISME J. 3, 738-744 (2009).

43. Girvan, M. S., Campbell, C. D., Killham, K., Prosser, J. I. \& Glover, L. A. Bacterial diversity promotes community stability and functional resilience after perturbation. Environ. Microbiol. 7, 301-313 (2005).

44. Allison, S. D. \& Martiny, J. B. H. Resistance, resilience, and redundancy in microbial communities. Proc. Natl Acad. Sci. USA 105, 11512-11519 (2008).

45. Malik, A. A., Thomson, B. C., Whiteley, A. S., Bailey, M. \& Griffiths, R. I. Bacterial physiological adaptations to contrasting edaphic conditions identified using landscape scale metagenomics. mBio 8, e00799-17 (2017).

46. Evans, S., Martiny, J. B. H. \& Allison, S. D. Effects of dispersal and selection on stochastic assembly in microbial communities. ISME J. 11, 176-185 (2017).

47. Vellend, M. et al. Assessing the relative importance of neutral stochasticity in ecological communities. Oikos 123, 1420-1430 (2014).

48. Hartl, D. L., Clark, A. G. \& Clark, A. G. Principles of Population Genetics. 116 (Sinauer associates Sunderland, MA, 1997).

49. Vellend, M. et al. Homogenization of forest plant communities and weakening of species-environment relationships via agricultural land use. J. Ecol. 95, 565-573 (2007).

50. Dini-Andreote, F., Stegen, J. C., van Elsas, J. D. \& Salles, J. F. Disentangling mechanisms that mediate the balance between stochastic and deterministic processes in microbial succession. Proc. Natl Acad. Sci. USA 112, 1326-1332 (2015).

51. Xun, W. et al. Environmental conditions rather than microbial inoculum composition determine the bacterial composition, microbial biomass and enzymatic activity of reconstructed soil microbial communities. Soil Biol. Biochem. 90, 10-18 (2015).

52. Fierer, N. Embracing the unknown: disentangling the complexities of the soil microbiome. Nat. Rev. Microbiol. 15, 579-590 (2017).

53. Coyte, K. Z., Schluter, J. \& Foster, K. R. The ecology of the microbiome: Networks, competition, and stability. Science 350, 663-666 (2015). 
54. Brophy, C. et al. Biodiversity and ecosystem function: making sense of numerous species interactions in multi-species communities. Ecology 98, 1771-1778 (2017).

55. Coates, K. D. \& Astrup, R. Competitive interactions across a soil fertility gradient in a multispecies forest. J. Ecol. 101, 806-818 (2013).

56. Cotrufo, M. F., Wallenstein, M. D., Boot, C. M., Denef, K. \& Paul, E. The microbial efficiency-matrix stabilization (MEMS) framework integrates plant litter decomposition with soil organic matter stabilization: do labile plant inputs form stable soil organic matter? Glob. Change Biol. 19, 988-995 (2013).

57. Fontaine, S., Mariotti, A. \& Abbadie, L. The priming effect of organic matter: a question of microbial competition? Soil Biol. Biochem. 35, 837-843 (2003).

58. Hassett, D. J. \& Cohen, M. S. Bacterial adaptation to oxidative stress: implications for pathogenesis and interaction with phagocytic cells. FASEB J. 3, 2574-2582 (1989).

59. Aislabie, J. \& Lloyd-Jones, G. A review of bacterial-degradation of pesticides. Soil Res. 33, 925-942 (1995).

60. Chave, J. Neutral theory and community ecology. Ecol. Lett. 7, 241-253 (2010).

61. Weimer, P. J. Why don't ruminal bacteria digest cellulose faster? J. Dairy Sci. 79, 1496-1502 (1996).

62. Rousk, J. et al. Soil bacterial and fungal communities across a $\mathrm{pH}$ gradient in an arable soil. ISME J. 4, 1340-1351 (2010).

63. Edgar, R. C. UPARSE: highly accurate OTU sequences from microbial amplicon reads. Nat. Methods 10, 996-998 (2013).

64. Peng, Y., Leung, H. C. M., Yiu, S. M. \& Chin, F. Y. L. IDBA - A practical iterative de bruijn graph de novo assembler. Lect. Notes Comput. Sci. 6044, 426-440 (2010).

65. Noguchi, H., Park, J. \& Takagi, T. MetaGene: prokaryotic gene finding from environmental genome shotgun sequences. Nucleic Acids Res. 34, 5623-5630 (2006).

66. Li, W. \& Godzik, A. Cd-hit: a fast program for clustering and comparing large sets of protein or nucleotide sequences. Bioinformatics 22, 1658-1659 (2006).

67. Gu, S., Fang, L. \& Xu, X. Using SOAPaligner for short reads alignment. Curr. Protoc. Bioinf. 44, 11.11. 1-11.11. 17 (2013).

68. Oksanen, J. F.et al. vegan: Community Ecology Package. R. package version 2.41 (2016).

69. Hamady, M., Lozupone, C. \& Knight, R. Fast UniFrac: facilitating highthroughput phylogenetic analyses of microbial communities including analysis of pyrosequencing and PhyloChip data. ISME J. 4, 17-27 (2010).

70. Kembel, S. W. et al. Picante: R tools for integrating phylogenies and ecology. Bioinformatics 26, 1463-1464 (2010).

71. Wang, J. et al. Phylogenetic beta diversity in bacterial assemblages across ecosystems: deterministic versus stochastic processes. ISME J. 7, 1310-1321 (2013).

72. Stegen, J. C., Lin, X., Konopka, A. E. \& Fredrickson, J. K. Stochastic and deterministic assembly processes in subsurface microbial communities. ISME J. 6, 1653-1664 (2012).

73. De Caceres, M. \& Jansen, F. Package 'indicspecies'. Website: http://th.archive. ubuntu.com/cran/web/packages/indicspecies/ (2016).

\section{Acknowledgements}

We thank Professor Pascal Simonet (Universite de Lyon), Professor Shuijin Hu (North Carolina State University), and Professor George A Kowalchuk (Utrecht University) for their valuable comments on this manuscript. This research was financially supported by the National Key Basic Research Program of China (973 program, 2015CB150500), the National Natural Science Foundation for Young Scientists of China (41601252), Special and General Financial Grant from the China Postdoctoral Science Foundation (2017T100379 and 2016M601833), the Young Elite Scientists Sponsorship Program by CAST (2018QNRC001), the Science and Technology Innovation Project of Chinese Academy of Agricultural Sciences, and the Fundamental Research Funds for the Central Universities (KJQN201748).

\section{Author contributions}

W.-B.X., R.Z., Z.X., N.Z., and Q.S. designed the study. W.L. and Y.R. performed experimental work and detailed the sampling. W.L., Y.L., and Y.M. conducted the DNA purification and organized the sequencing. W.-B.X. and W.X. carried out the bioinformatics and statistical analysis. W.-B.X. and R.Z. created the figures and drafted the manuscript. All authors helped review, edit, and complete the manuscript.

\section{Additional information}

Supplementary Information accompanies this paper at https://doi.org/10.1038/s41467 019-11787-5.

Competing interests: The authors declare no competing interests.

Reprints and permission information is available online at http://npg.nature.com/ reprintsandpermissions/

Peer review information: Nature Communications thanks Binu Mani Tripathi and the other, anonymous, reviewer(s) for their contribution to the peer review of this work. Peer reviewer reports are available.

Publisher's note: Springer Nature remains neutral with regard to jurisdictional claims in published maps and institutional affiliations.

Open Access This article is licensed under a Creative Commons Attribution 4.0 International License, which permits use, sharing, adaptation, distribution and reproduction in any medium or format, as long as you give appropriate credit to the original author(s) and the source, provide a link to the Creative Commons license, and indicate if changes were made. The images or other third party material in this article are included in the article's Creative Commons license, unless indicated otherwise in a credit line to the material. If material is not included in the article's Creative Commons license and your intended use is not permitted by statutory regulation or exceeds the permitted use, you will need to obtain permission directly from the copyright holder. To view a copy of this license, visit http://creativecommons.org/ licenses/by/4.0/

(C) The Author(s) 2019 